\title{
ON MULTICOLOR RAMSEY NUMBERS FOR LOOSE $k$-PATHS OF LENGTH THREE
}

\author{
TOMASZ ŁUCZAK, JOANNA POLCYN, AND ANDRZEJ RUCIŃSKI
}

\begin{abstract}
We show that there exists an absolute constant $A$ such that for each $k \geq 2$ and every coloring of the edges of the complete $k$-uniform hypergraph on $A r$ vertices with $r$ colors, one of the color classes contains a loose path of length three.
\end{abstract}

\section{INTRODUCTION}

For $k \geq 2$, a $k$-uniform hypergraph (or, briefly, a $k$-graph) is an ordered pair $H=(V, E)$, where $V=V(H)$ is a finite set and $E=E(H)$ is a subset of the set $\left(\begin{array}{l}V \\ k\end{array}\right)$ of all $k$-element subsets of $V$. If $E=\left(\begin{array}{l}V \\ k\end{array}\right)$, we call $H$ complete and denote it by $K_{n}^{(k)}$, where $n=|V|$. The elements of $V$ and $E$ are called, respectively, the vertices and edges of $H$. We often identify $H$ with $E(H)$, writing, for instance, $|H|$ instead of $|E(H)|$. The degree $\operatorname{deg}_{H}(v)$ of a vertex $v$ in $H$ equals the number of edges of $H$ which contain $v$. A star is a $k$-graph $S$ with a vertex $v$ contained in all the edges of $S$. A star is full if it consists of all sets in $\left(\begin{array}{l}V \\ k\end{array}\right)$ containing $v$, that is, in which $\operatorname{deg}_{S}(v)=\left(\begin{array}{l}n-1 \\ k-1\end{array}\right)$.

Let $P^{(k)}$ denote a loose $k$-uniform path (shortly, a $k$-path) of length three, that is, the only connected $k$-graph on $3 k-2$ vertices with three edges and no vertex of degree three. In this paper we study the multicolor Ramsey number $R\left(P^{(k)} ; r\right)$ for $P^{(k)}$ defined as the smallest number $n$ such that each coloring of the edges of the complete $k$-graph $K_{n}^{(k)}$ with $r$ colors leads to a monochromatic copy of $P^{(k)}$. In the graph case, i.e. when $k=2$, it is easy to see that the value of $R(P ; r)$ is equal to $2 r+c_{r}$, where $c_{r} \in\{0,1,2\}$ and depends on the divisibility of $r$ by three (see [10]). On the other hand, coloring the edges of $K_{r+3 k-4}^{(k)}$ by $r-1$ stars and one clique $K_{3 k-3}^{(k)}$ (e.g., [3], Proposition 3.1) shows that

$$
R\left(P^{(k)} ; r\right) \geq r+3 k-3 \text {. }
$$

Date: March 18, 2017.

2010 Mathematics Subject Classification. Primary: 05D10, secondary: 05C38, 05C55, $05 \mathrm{C} 65$.

Key words and phrases. Ramsey number, hypergraphs, paths.

The first author supported by Polish NSC grant 2012/06/A/ST1/00261. The third author supported by the Polish NSC grant 2014/15/B/ST1/01688. 
It is conjectured that for each $k \geq 3$ and all $r$ there is equality in (1). So far, it has been verified only for $k=3$ and $r=2,3, \ldots, 10(2$, 3, 4, 9, 8]). In fact, for $k=3$ and $r=2$ the Ramsey number has been determined for paths of all lengths [7].

A general upper bound on $R\left(P^{(k)} ; r\right), k \geq 3$, follows by a standard application of Turán numbers. Indeed, it was proved by Füredi, Jiang, and Seiver [1] that for $n \geq n_{0}(k)$ the unique largest $P^{(k)}$-free $k$-graph on $n$ vertices is a full star which contains at most $\left(\begin{array}{l}n-1 \\ k-1\end{array}\right)$ edges. From this it follows that for $r$ large enough $R\left(P^{(k)} ; r\right) \leq k r+1$ and if we use the fact that the extremal graph is unique we get (see [3], Proposition 3.2)

$$
R\left(P^{(k)} ; r\right) \leq k r,
$$

valid for all $k \geq 3$ and $r \geq r_{0}(k)$. Similar results for loose cycles of length three were obtained by Gyárfás and Raeisi [2]. For $k=3$, it was proved by Łuczak and Polcyn [6] that $R\left(P^{(3)} ; r\right) \leq 2 r+O(\sqrt{r})$. The main goal of this paper is to show that for $r$ large enough $R\left(P^{(k)} ; r\right) / r$ is bounded from above by a constant which does not depend on $k$.

Theorem 1. For each $k \geq 3$ there exists $r_{k}$ such that for all $r \geq r_{k}$

$$
R\left(P^{(k)} ; r\right) \leq 250 r \text {. }
$$

\section{Proof of Theorem 1}

In view of (2), we may restrict ourselves to $k \geq 250$. Our proof uses two results on Turán numbers for loose $k$-paths of length two and three. The first of them was proved by Keevash, Mubayi, and Wilson [5].

Lemma 2. Let $k \geq 4$ and $H$ be a k-uniform hypergraph on $n$ vertices in which no two edges intersect on a single vertex. Then, for large $n$, $|H| \leq\left(\begin{array}{l}n-2 \\ k-2\end{array}\right)$.

The second result, due to Füredi, Jiang, and Seiver [1], deals with the main object of our study: $P^{(k)}$, the loose $k$-uniform path of length three.

Lemma 3. Let $k \geq 3$ and $H$ be a $P^{(k)}$-free $k$-uniform hypergraph on $n$ vertices. Then, for large $n,|H| \leq\left(\begin{array}{l}n-1 \\ k-1\end{array}\right)$.

It is also proved in [1] that, in fact, the only extremal $k$-graph is a full star. Theorem 1 is a direct consequence of the following 'stability' version of Lemma 3 which states, roughly, that the structure of each $P^{(k)}$-free dense $k$-graph is dominated by a giant star.

Lemma 4. For every $k \geq 250$ and $n \geq n_{0}(k)$, each $P^{(k)}$-free $k$-uniform hypergraph $H$, has a vertex $v$ with degree

$$
\operatorname{deg}_{H}(v) \geq|H|-0.96^{k}\left(\begin{array}{l}
n-1 \\
k-1
\end{array}\right) .
$$


We defer the proof of Lemma 4 to the next section. Here we show how Theorem 1 follows from it.

Proof of Theorem 1. For a given $k \geq 250$ and $A=250$ let $r \geq r_{k}$, where $r_{k}$ is chosen so that $250 r_{k} \geq n_{0}(k)$ with $n_{0}(k)$ defined as in Lemma 4. Suppose that the complete $k$-graph $K:=K_{A r}^{(k)}$ on $A r$ vertices is colored with colors $1,2, \ldots, r$ in such a way that no monochromatic $P^{(k)}$ emerges. For every color $c$ choose (possibly with repetitions) a vertex $v_{c}$ with maximum degree in this color and let $R=\left\{v_{c}: c=\right.$ $1,2, \ldots, r\}$.

Consider now the complete $k$-graph $H$ obtained from $K$ by removing all vertices in $R$. We have $|V(H)| \geq A r-|R| \geq(A-1) r$ and thus $|E(H)| \geq\left(\begin{array}{c}(A-1) r \\ k\end{array}\right)$. On the other hand, by applying Lemma 4 to each color class, we have $|H| \leq r(0.96)^{k}\left(\begin{array}{c}A r-1 \\ k-1\end{array}\right)$. On the other hand, since $k \geq A=250$, we have

$$
r(0.96)^{k}\left(\begin{array}{c}
A r-1 \\
k-1
\end{array}\right)<\left(\begin{array}{c}
(A-1) r \\
k
\end{array}\right)
$$

a contradiction. To see (3), observe first that the two sides of (3) are asymptotic (as $r$ is growing) to, respectively, $0.96^{k} A^{k-1} r^{k} /(k-1)$ ! and $(A-1)^{k} r^{k} / k$ ! . Thus it remains to show that $(A-1)^{k}>k(0.96)^{k} A^{k-1}$, or, equivalently, $(A-1)(1-1 / A)^{k-1}>k(0.96)^{k}$. Now it is enough to observe that $1-1 / A \geq 0.99$ for $A \geq 250$ and $k<(99 / 96)^{k}$ for $k \geq 167$.

\section{Proof of Lemma 4}

We begin by stating two elementary facts the short proofs of which are provided for completeness.

Fact 5. Every hypergraph $H$ contains a sub-hypergraph $G$ with minimum degree greater than $\frac{|E(H)|}{|V(H)|}$.

Proof. Define $G$ as a subhypergraph of $H$ which maximizes the ratio $\frac{|E(G)|}{|V(G)|}$ and has the smallest number of vertices. If for some $v \in V(G)$, $\operatorname{deg}_{G}(v) \leq \frac{|E(H)|}{|V(H)|}$, then

$$
\frac{|E(G-v)|}{|V(G-v)|} \geq \frac{|E(G)|-|E(H)| /|V(H)|}{|V(G)|-1} \geq \frac{|E(G)|}{|V(G)|},
$$

which contradicts our choice of $G$.

Fact 6. Every bipartite graph $B$ with vertex classes $V_{1}$ and $V_{2}$ contains a subgraph $G$ with $\operatorname{deg}_{G}(v) \geq|B| /\left(2\left|V_{i}\right|\right)$ for every vertex $v \in V(G) \cap V_{i}$, $i=1,2$.

Proof. Let us remove one by one the vertices with (current) degree smaller than the above bounds. Then, by the time the degrees of all 
remaining vertices satisfy the required bounds, we remove fewer than

$$
\left|V_{1}\right| \times|B| /\left(2\left|V_{1}\right|\right)+\left|V_{2}\right| \times|B| /\left(2\left|V_{2}\right|\right)=|B|
$$

edges, and so the final subgraph $G$ is non-empty.

Lemma 4is a straightforward consequence of the following two propositions.

Proposition 7. For all $k \geq 3, b>0$, and sufficiently large $n$, the following holds. Let $H$ be a $P^{(k)}$-free, $n$-vertex, $k$-uniform hypergraph and let, for some $v \in V(H), \operatorname{deg}_{H}(v) \geq b\left(\begin{array}{l}n-1 \\ k-1\end{array}\right)$. Then,

$$
\operatorname{deg}_{H}(v) \geq|H|-\left(1-\left(\frac{b}{k-1}\right)^{1 /(k-2)}\right)^{k-1}\left(\begin{array}{l}
n-1 \\
k-1
\end{array}\right) .
$$

Proof. Let $H(v)$ be the link of $v$ in $H$, that is, the $(k-1)$-uniform, $(n-1)$-vertex hypergraph consisting of all $(k-1)$-element subsets of $V(H)$ which together with $v$ form edges in $H$. Note that $|H(v)|=$ $\operatorname{deg}_{H}(v)$. Fact 5 implies that there is a subgraph $F$ of $H(v)$ with minimum degree

$$
\delta(F) \geq \delta:=\frac{b}{k-1}\left(\begin{array}{l}
n-2 \\
k-2
\end{array}\right) .
$$

Claim 8. The number of vertices $|V(F)|$ of $F$ is bounded from below by

$$
|V(F)| \geq\left(\frac{b}{k-1}\right)^{1 /(k-2)}(n-1) .
$$

Proof. Since

$$
\left(\begin{array}{c}
|V(F)| \\
k-1
\end{array}\right) \geq|F| \geq|V(F)| \frac{b}{(k-1)^{2}}\left(\begin{array}{c}
n-2 \\
k-2
\end{array}\right),
$$

it follows that

$$
\left(\begin{array}{c}
|V(F)|-1 \\
k-2
\end{array}\right) \geq \frac{b}{k-1}\left(\begin{array}{l}
n-2 \\
k-2
\end{array}\right)
$$

so,

$$
1 \geq \frac{b}{k-1} \frac{(n-2)_{k-2}}{(|V(F)|-1)_{k-2}}>\frac{b}{k-1}\left(\frac{n-1}{|V(F)|}\right)^{k-2},
$$

which implies the required bound for $|V(F)|$.

Claim 9. Let $n$ be sufficiently large. For every edge $e \in H$, either $v \in e$ or $e \cap V(F)=\emptyset$.

Proof. Suppose there exists an edge $e \in H$ such that $v \notin e$ and $e \cap$ $V(F) \neq \emptyset$. Let $w \in e \cap V(F)$. Since $\operatorname{deg}_{F}(w) \geq \delta=\Omega\left(n^{k-2}\right)$ while the number of edges of $F$ intersecting $e$ on at least two vertices is $O\left(n^{k-3}\right)$, there is an edge $f^{\prime} \in F$ such that $e \cap f^{\prime}=\{w\}$. Further, since $\operatorname{deg}_{H}(v) \geq b\left(\begin{array}{l}n-1 \\ k-1\end{array}\right)$ while the number of edges of $H$ containing $v$ 
and intersecting $e \cup f^{\prime}$ is $O\left(n^{k-2}\right)$, there is an edge $h \in H$ such that $v \in h$ and $h \cap\left(e \cup f^{\prime}\right)=\emptyset$. The edges $e, f^{\prime} \cup\{v\}$, and $h$ form a copy of $P^{(k)}$ in $H$, a contradiction.

In view of Claim 9, to complete the proof of Proposition 7, we bound from above the number of edges of $H$ which do not contain $v$ by $\mid H-$ $V(F) \mid$, where $H-V(F)$ is the induced subhypergraph of $H$ obtained by deleting all vertices of $F$. Since $H$, and thus $H-V(F)$, is $P^{(k)}$ free, we can bound $|H-V(F)|$ by the Turán number for $P^{(k)}$ given in Lemma 3. Using the bound for $|V(F)|$ given by Claim 8, we get

$$
\begin{aligned}
|H-V(F)| & \leq\left(\begin{array}{c}
n-|V(F)|-2 \\
k-1
\end{array}\right) \\
& \leq\left(\begin{array}{c}
n-(n-1)(b /(k-1))^{1 /(k-2)}-2 \\
k-1
\end{array}\right) \\
& <\left(\begin{array}{c}
(n-1)\left(1-(b /(k-1))^{1 /(k-2)}\right) \\
k-1
\end{array}\right) \\
& \leq\left(1-\left(\frac{b}{k-1}\right)^{1 /(k-2)}\right)^{k-1}\left(\begin{array}{c}
n-1 \\
k-1
\end{array}\right) .
\end{aligned}
$$

Proposition 10. For all $k \geq 250$ and sufficiently large $n$ the following holds. If $H$ is a $P^{(k)}$-free $k$-graph on $n$ vertices and $|H| \geq 0.96^{k}\left(\begin{array}{c}n-1 \\ k-1\end{array}\right)$, then $\Delta(H) \geq 0.9^{k}\left(\begin{array}{l}n-1 \\ k-1\end{array}\right)$.

Proof. Let $H$ be a $P^{(k)}$-free $k$-graph on $n$ vertices and with $|H| \geq$ $0.96^{k}\left(\begin{array}{l}n-1 \\ k-1\end{array}\right)$. By $F$ we denote the shadow of $H$, i.e.

$$
F=\left\{f \in[n]^{k-1}: f \subset e \text { for some } e \in H\right\} .
$$

Let us now suppose that $\Delta(H)<0.9^{k}\left(\begin{array}{l}n-1 \\ k-1\end{array}\right)$. We shall show that this assumption leads to a contradiction.

The main idea of the argument goes roughly as follows. First we deal with the case when $F$ is small (Claim 11 below). Then there are many vertices $v$ with large links. Consequently, it is enough to find in $F$ a loose $(k-1)$-path of length two, say $f_{1}, f_{2}$ (and for that, due to Lemma 2 we only require that $\left.|F|=\Omega\left(n^{k-3}\right)\right)$ and find another $f_{3}$ in $F$ so that $\left(f_{1} \cup f_{2}\right) \cap f_{3}=\emptyset$. Then, for some $v_{1}, v_{2} \in V(H)$, the edges $\left\{v_{1}\right\} \cup f_{1},\left\{v_{2}\right\} \cup f_{2}$, and $\left\{v_{2}\right\} \cup f_{3}$ form a $P^{(k)}$ in $H$. In the case $F$ is large we select the three disjoint subsets of vertices, $W_{1}, W_{2}$ and $W_{3}$, such that the unions $S_{i}$ of the links of vertices in $W_{i}$ are edge-disjoint and roughly of the same size, for each $i=1,2,3$. Since $F$ is large, so are all $S_{i}$ 's; in fact each of them covers a majority of the vertices of $H$. Thus, one can find a $(k-1)$-path of length three consisting of some sets $f_{1}, f_{2}, f_{3}$, where $f_{i} \in S_{i}$ for $i=1,2,3$, which in turn, can be easily extended to $P^{(k)}$. 
In order to make the above precise, let us start with the following observation.

Claim 11. $|F| \geq \frac{1}{4}|H|$.

Proof. Let us consider an auxiliary bipartite graph $B$, with vertex classes $V(H)$ and $F$, and with edge set

$$
\{\{v, f\}:\{v\} \cup f \in H\} .
$$

Clearly, $|B|=k|H|$. Further, define

$$
F^{\prime}=\{f \in F:|\{e \in H: f \subset e\}| \geq 2 k\}
$$

and observe that $\left|F^{\prime}\right| \leq\left(\begin{array}{l}n-1 \\ k-2\end{array}\right)$. Indeed, otherwise, by the Turán number for $P^{(k-1)}, F^{\prime}$ would contain a copy of $P^{(k-1)}$ which could be easily extended to a copy of $P^{(k)}$ in $H$.

Let $B^{\prime}$ be the subgraph of $B$ consisting of all edges with one endpoint in $F^{\prime}$. We have

$$
|B|=\sum_{f} \operatorname{deg}_{B}(f) \leq\left|B^{\prime}\right|+\left(|F|-\left|F^{\prime}\right|\right) 2 k,
$$

SO

$$
|F| \geq|F|-\left|F^{\prime}\right| \geq \frac{1}{2 k}\left(|B|-\left|B^{\prime}\right|\right) .
$$

Thus, recalling that $|B|=k|H|$, to complete the proof of Claim 11, it suffices to show that

$$
\left|B^{\prime}\right| \leq|B| / 2 \text {. }
$$

Suppose that $\left|B^{\prime}\right| \geq|B| / 2$. Then,

$$
\left|B^{\prime}\right| \geq \frac{k}{2}|H| \geq \frac{k}{2}(0.96)^{k}\left(\begin{array}{l}
n-1 \\
k-1
\end{array}\right) .
$$

We apply Fact 7 to $B^{\prime}$, obtaining a subgraph $B^{\prime \prime}$ with vertex sets $V_{1} \subset$ $V(H)$ and $F^{\prime \prime} \subset F^{\prime}$ such that each vertex $v \in V_{1}$ has in $B^{\prime \prime}$ degree at least $\frac{1}{4}(0.96)^{k}\left(\begin{array}{c}n-1 \\ k-2\end{array}\right)$ and each $f \in F^{\prime \prime}$ has in $B^{\prime \prime}$ degree at least $\frac{1}{4}(0.96)^{k} n$.

Since, for large $n,\left|F^{\prime \prime}\right| \geq \frac{1}{4}(0.96)^{k}\left(\begin{array}{l}n-1 \\ k-2\end{array}\right)>\left(\begin{array}{l}n-2 \\ k-3\end{array}\right)$, by Lemma 2, $F^{\prime \prime}$ contains two $(k-1)$-sets $f_{1}, f_{2}$ such that $\left|f_{1} \cap f_{2}\right|=1$. Let $N_{i}$ be the neighborhood of $f_{i}$ in $B^{\prime \prime}, i=1,2$. If there was an edge $(v, f) \in B^{\prime \prime}$ with $v \in N_{1} \cup N_{2}$ (say, $\left.v \in N_{2}\right)$ and $\left(f_{1} \cup f_{2}\right) \cap f=\emptyset$, then the $k$-sets $\left\{v_{1}\right\} \cup f_{1}$, $\{v\} \cup f_{2}$, and $\{v\} \cup f$, where $v_{1} \in N_{1}, v_{1} \neq v$, would form a copy of $P^{(k)}$ in $H$, a contradiction. Thus, in $B^{\prime \prime}$, all neighbors $f$ of vertices in $N_{1} \cup N_{2}$ must intersect $f_{1} \cup f_{2}$. Since $\left|N_{1} \cup N_{2}\right| \geq\left|N_{1}\right| \geq \frac{1}{4}(0.96)^{k} n$, the number of edges of $B^{\prime \prime}$ leaving $N_{1} \cup N_{2}$ is at least

$$
\frac{1}{4}(0.96)^{k} n \times \frac{1}{4} 0.96^{k}\left(\begin{array}{l}
n-1 \\
k-2
\end{array}\right)=\frac{1}{16}(0.96)^{2 k} n\left(\begin{array}{l}
n-1 \\
k-2
\end{array}\right) .
$$


Each of these edges of $B^{\prime \prime}$ represents an edge of $H$ which intersects $f_{1} \cup f_{2}$, a set of size smaller than $2 k$. Hence, by averaging, there exists a vertex in $f_{1} \cup f_{2}$ belonging to at least

$$
\frac{1}{32 k}(0.96)^{2 k} n\left(\begin{array}{l}
n-1 \\
k-2
\end{array}\right)>0.9^{k}\left(\begin{array}{l}
n-1 \\
k-1
\end{array}\right)
$$

of these edges (note that the last inequality is valid for $k \geq 250$ ). This contradicts our assumption on $\Delta(H)$ and, therefore, completes the proof of Claim [11.

To continue with the proof of Proposition [10, for every $f \in F$ we choose just one vertex $v_{f}$ such that $\left\{v_{f}\right\} \cup f \in H$. Observe that by our assumption on $\Delta(H)$, for each $v \in V(H)$,

$$
\left|\left\{f \in F: v=v_{f}\right\}\right|<0.9^{k}\left(\begin{array}{l}
n-1 \\
k-1
\end{array}\right) .
$$

Further, we split the vertex set $V(H)$ randomly into two parts, $U_{1}$ and $U_{2}$, where each vertex belongs to $U_{1}$ independently with probability $1 / k$. We call a set $f \in F$ proper if $v_{f} \in U_{1}$ and $f \subseteq U_{2}$.

Let $X$ count the number of proper sets. Since

$$
P(f \text { is proper })=\frac{1}{k} \cdot\left(\frac{k-1}{k}\right)^{k-1} \geq \frac{1}{k} \cdot \frac{1}{e}>\frac{1}{3 k},
$$

by Claim 11,

$$
E X=\sum_{f \in F} P(f \text { is proper })=|F| \cdot P(f \text { is proper }) \geq \frac{0.96^{k}}{12 k}\left(\begin{array}{l}
n-1 \\
k-1
\end{array}\right) .
$$

Thus, there exists a partition $\left(U_{1}, U_{2}\right)$ such that the number of proper sets $f$ is at least $\frac{0.96^{k}}{12 k}\left(\begin{array}{l}n-1 \\ k-1\end{array}\right)$. For each $v \in U_{1}$, set

$$
F_{v}=\left\{f \in F: v=v_{f} \text { and } f \subset U_{2}\right\} \quad \text { and } \quad \phi_{v}=\frac{\left|F_{v}\right|}{\left(\begin{array}{c}
n-1 \\
k-1
\end{array}\right)} .
$$

By (51) and the above lower bound on the number of proper sets $f$, we have $\sum_{v \in U_{1}} \phi_{v}>0.96^{k} /(12 k)$ and, for each $v$, we have also $\phi_{v}<0.9^{k}$. We partition the set $\left\{v \in U_{1}: F_{v} \neq \emptyset\right\}$ into three subsets $W_{1}, W_{2}, W_{3}$ so that the sums $S_{i}:=\sum_{v \in W_{i}} \phi_{v}, i=1,2,3$, are as close to each other as possible. This can be done, for instance, by a greedy algorithm which places the vertices one after another into the set with the current minimum total of $\phi_{v}$ 's. Then, assuming that $S_{1} \leq S_{2} \leq S_{3}$, we have

$$
S_{1}>S_{3}-0.9^{k} \geq \frac{1}{3}\left(S_{1}+S_{2}+S_{3}\right)-0.9^{k} \geq \frac{1}{4}\left(S_{1}+S_{2}+S_{3}\right),
$$

provided

$$
0.9^{k}<\frac{1}{12} \times \frac{1}{12 k} 0.96^{k}=\frac{1}{144 k} 0.96^{k} \leq \frac{1}{12}\left(S_{1}+S_{2}+S_{3}\right),
$$


which is valid for $k \geq 250$. Hence, for each $i=1,2,3$,

$$
\sum_{v \in W_{i}}\left|F_{v}\right| \geq \frac{0.96^{k}}{48 k}\left(\begin{array}{l}
n-1 \\
k-1
\end{array}\right)
$$

The sets $W_{1}, W_{2}, W_{3}$ generate a corresponding partition of the proper sets $f$ into 'colors' $C_{i}=\bigcup_{v \in W_{i}} F_{v}$. In order to complete the proof of Proposition 10, it suffices to show that such a 3 -coloring contains a $(k-1)$-path of length three whose edges are colored with different colors. Such a path can be extended to a copy of $P^{(k)}$ in $H$, yielding a contradiction.

Howeover, all sets $W_{i}$ are so dense that the existence of such a path is an easy consequence of Fact 5. Indeed, recall that in each color there are at least $0.96^{k} /(48 k)\left(\begin{array}{l}n-1 \\ k-1\end{array}\right)$ edges. Therefore, by Fact 5 , in each color $C_{i}, i=1,2,3$, viewed as a $(k-1)$-graph, one can find a sub-hypergraph $G_{i}$ with

$$
\delta\left(G_{i}\right) \geq \frac{0.96^{k}}{48 k^{2}}\left(\begin{array}{l}
n-2 \\
k-2
\end{array}\right) .
$$

Moreover, $\left|V\left(G_{i}\right)\right| \geq 0.9 n$, since otherwise for each vertex $v \in V\left(G_{i}\right)$,

$$
\operatorname{deg}_{G_{i}}(v) \leq\left(\begin{array}{c}
0.9 n \\
k-2
\end{array}\right)<\frac{0.9^{k-2} n^{k-2}}{(k-2) !}<\frac{0.96^{k}}{48 k^{2}}\left(\begin{array}{c}
n-2 \\
k-2
\end{array}\right) \leq \delta_{G_{i}},
$$

where the penultimate inequality holds for $k \geq 250$. Consequently, the intersection of the vertex sets of these three graphs, $U:=V\left(G_{1}\right) \cap$ $V\left(G_{2}\right) \cap V\left(G_{3}\right)$, has size $|U| \geq 0.7 n$.

Fix a vertex $v \in U$. Since $\operatorname{deg}_{G_{1}}(v) \geq \frac{0.96^{k}}{48 k^{2}}\left(\begin{array}{c}n-2 \\ k-2\end{array}\right)$ and the number of edges of $G_{1}$ with $f \cap U=\{v\}$ is at most $\left(\begin{array}{l}0.3 n \\ k-2\end{array}\right)$, there exists an edge $f_{1} \in$ $G_{1}$ and a vertex $w \in U, w \neq v$, such that $\{v, w\} \subset f_{1} \cap U$. Moreover, since the number of edges of $G_{2}$ containing $v$ and another vertex of $f_{1}$ is $O\left(n^{k-3}\right)$, we can find $f_{2} \in G_{2}$ such that $f_{1} \cap f_{2}=\{v\}$. Similarly, there exists $f_{3} \in G_{3}$ such that $f_{3} \cap\left(f_{1} \cup f_{2}\right)=\{w\}$. Then the edges $f_{2}, f_{1}$, and $f_{3}$ form a desired copy of $P^{(k-1)}$ in $F$. Finally, the edges $\left\{v_{f_{i}}\right\} \cup f_{i}, i=1,2,3$, create a $k$-path $P^{(k)}$ in $H$, a contradiction.

Proof of Lemma 4. If $|H|<0.96^{k}\left(\begin{array}{l}n-1 \\ k-1\end{array}\right)$ then the assertion obviously holds. Let us assume that $|H| \geq 0.96^{k}\left(\begin{array}{l}n-1 \\ k-1\end{array}\right)$. Then, by Proposition 10, there exists a vertex $v \in V(H)$ with

$$
\operatorname{deg}_{H}(v) \geq 0.9^{k}\left(\begin{array}{l}
n-1 \\
k-1
\end{array}\right) .
$$

Therefore, by Proposition 7 with $b=0.9^{k}$,

$$
\operatorname{deg}_{H}(v) \geq|H|-\left(1-\left(\frac{0.9^{k}}{k-1}\right)^{\frac{1}{k-2}}\right)^{k-1}\left(\begin{array}{l}
n-1 \\
k-1
\end{array}\right) .
$$


Thus, all we need to verify is that

$$
\left(1-\left(\frac{0.9^{k}}{k-1}\right)^{\frac{1}{k-2}}\right)^{k-1}<0.96^{k} .
$$

To this end, observe that

$$
0.96^{k /(k-1)}>0.96^{2}>0.9,
$$

while

$$
1-\left(\frac{0.9^{k}}{k-1}\right)^{\frac{1}{k-2}}<0.9
$$

is equivalent to

$$
0.1^{k-2}(k-1)<0.9^{k}
$$

which holds for $k \geq 3$.

\section{REFERENCES}

[1] Z. Füredi, T. Jiang, R. Seiver, Exact solution of the hypergraph Turán problem for k-uniform linear paths, Combinatorica 34(3) (2014) 299-322

[2] A. Gyárfás, G. Raeisi, The Ramsey number of loose triangles and quadrangles in hypergraphs, Electron. J. Combin., 19(2) (2012), \# R30.

[3] E. Jackowska, The 3-color Ramsey number for a 3-uniform loose path of length 3, Australasian J. Combin, 63(2) (2015), 314-320.

[4] E. Jackowska, J. Polcyn, A. Ruciński, Multicolor Ramsey numbers and restricted Turán numbers for the loose 3-uniform path of length three, arXiv:1506.03759v1, submitted.

[5] P. Keevash, D. Mubayi, R. M. Wilson, Set systems with no singleton intersection, SIAM J. Discrete Math. 20 (2006), 1031-1041.

[6] T. Luczak, J. Polcyn, On the multicolor Ramsey number for 3-paths of length three, Electron. J. Combin., 24(1) (2017), \#P1.27.

[7] G.R. Omidi, M. Shahsiah, Ramsey numbers of 3-uniform loose paths and loose cycles, J. Comb. Theory, Ser. A, 121 (2014), 64-73.

[8] J. Polcyn, One more Turán number and Ramsey number for the loose 3uniform path of length three, Discuss. Math. Graph Theory, 37 (2017) 443-464.

[9] J. Polcyn, A. Ruciński, Refined Turán numbers and Ramsey numbers for the loose 3-uniform path of length three, Discrete Mathematics, 340 (2017) 107118.

[10] S.P. Radziszowski, Small Ramsey numbers, Electron. J. Combin., Dynamic Survey, DS\#1

Adam Mickiewicz University, Faculty of Mathematics and Computer

Science ul. Umultowska 87, 61-614 Poznań, Poland

E-mail address: tomasz@amu.edu.pl

Adam Mickiewicz University, Faculty of Mathematics and Computer

Science ul. Umultowska 87, 61-614 Poznań, Poland

E-mail address: joaska@amu.edu.pl

Adam Mickiewicz University, Faculty of Mathematics and Computer

Science ul. Umultowska 87, 61-614 Poznań, Poland

E-mail address: rucinski@amu.edu.pl 